\title{
16083
}

\section{ADIABATIC TRANSPORT IN THE FRACTIONAL QUANTUM HALL EFFECT REGIME}

\author{
Carlo W.J. Beenakker \\ Philips Research Laboratories \\ P.O. Box 80000 \\ 5600 JA Eindhoven, The Netherlands
}

\section{Introduction}

The quantum Hall effect (QHE) is the phenomenon that the Hall conductance $G_{H}$ is quantized in units of $e^{2} / h$, as expressed by the formula

$$
G_{H}=\frac{p}{q} \frac{e^{2}}{h}
$$

( $p$ and $q$ being mutually prime integers). The integer QHE $(q=1)$ was discovered 10 years ago by von Klitzing, Dorda, and Pepper [1] in the two-dimensional electron gas (2DEG) confined to a Si inversion layer. The fractional QHE ( $q>1$ and odd) was first observed by Tsui, Störmer, and Gossard [2] in the 2 DEG at the interface of a $\mathrm{Al}_{x} \mathrm{Ga}_{1-x} \mathrm{As} / \mathrm{GaAs}$ heterostructure. Microscopically the two effects are entirely different. The integer QHE, on the one hand, can be explained satisfactorily in terms of the states of non-interacting electrons in a magnetic field (the Landau levels). The fractional QHE, on the other hand, exists only because of electron-electron interactions [3]. Phenomenologically, however, the integer and fractional QHE are quite similar. In an unbounded 2 DEG this similarity is understood from Laughlin's general argument [4] that: (1) The Hall conductance shows a plateau as a function of magnetic field (or Fermi energy) whenever the quasi-particle excitations in the bulk of the 2 DEG are localized by disorder; and that: (2) The value of $G_{H}$ on the plateau is precisely an integer multiple $p$ of $e e^{*} / h$, where $e^{*}=e / q$ is the quasi-particle charge. (The product $e e^{*}$ appears because one $e$ is needed to change the unit of conductance from Amperes per electron Volts to Amperes per Volts). Theory and experiment on the QHE in an unbounded 2 DEG have been reviewed in the books by Prange and Girvin [5] and by Chakraborty and Pietiläinen [6] (see also the article by MacDonald in the present volume).

In the past few years, a variety of experiments have uncovered a novel phenomenology of the QHE on short length scales. For example, in small sub-micron-size samples 
the QHE can occur in the absence of disorder [7,8] and can show deviations from precise quantization [9]. An anomalous quantization of the Hall conductance has been observed [10] in samples which are large but which contain a pair of closely spaced current and voltage contacts: quantization of $G_{H}$ then occurs at multiples of $e^{2} / h$ detcrmined by the properties of the contacts, rather than of the bulk 2 DEG. Indeed, it has been possible in such an experiment to measure the fractional QHE in a 2 DEG which by conventional measurements shows the integer effect [11].

These anomalies are not easily understood within the conventional description of the QIIE, which determines the quantized value of $G_{H}$ from the charge of a quasi-particle excitation localized in the bulk of the 2 DEG. One necds a description which can be applied to small samples without disorder and which explicitly includes the properties of the current and voltage contacts. For the integer QIIE the Landauer-Büttiker formalism provides such a description [12]. A central concept in this formulation is the concept of an edge channel, which is the collection of states at the Fermi energy within a given Landau level. These states are extended along the cdges of the 2 DEG whencver the Fermi level lies between two Landau levels in the bulk. Many of the anomalies in the integer QIIE can be understood as resulting from the absence of local equilibrium at the edge, which in turn is a consequence of the reduction of scattering between edge channels in a strong magnetic field $[10,13,14]$. On short length scales the electron transport becomes fully adiabatic, i.e. without inter-edge channel scattering. Edge channels in the integer QIIE are defined in one-to-one correspondence with bulk Landau levcls. This singlc-electron description is not applicable to the fractional QHE, which is fundamentally a many-body effect. In this article we review recent work towards a generalization of the concept of adiabatic transport in edge channels, with the aim of providing a unified description of anomalies in the integer and fractional QIIE.

We first summarize, in section 2, the Landauer-Büttiker formalism for the integer QHE. Our generalization [15] to the fractional QHE is described in section 3 and applied to experiments in section 4. Two open problems are addressed in section 5 . One is the question: "What charge does the resistance measure?" The other refers to an alternative generalized Landauer formula proposed by MacDonald [16]. We will argue that the appearance of both "electron" and "hole" channels in this formula implies a novel limitation to the accuracy of the fractional QHE. Much of the material in the present article is based on a review with a wider scope written in collaboration with $\mathrm{H}$. van IIouten [17].

\section{Integer Edge Channels}

The Landaucr-Büttiker formalism $[18,19]$ is a linear response formalism which expresses the conductance (a non-equilibrium property) in terms of an equilibrium Fermi level property of the conductor. That property consists of a rational function of transmission probabilities between current and voltage contacts of propagating modes with the Fermi energy. In a strong magnetic field in the QHE regime the propagating modes are extended along the edges of the conductor, because all Fermi level states in the bulk are localized. The Landaucr-Büttiker formalism thus describes the integer QHE in terms of properties of edge states. We review this description in the present section.

We restrict the discussion to the case of a smoothly varying potential energy landscape $V(x, y)$ in the $2 \mathrm{DEG}$. The smoothness criterion is that $V$ should vary by less than the Landau level separation $\hbar \omega_{c} \equiv \hbar e B / m$ over a magnetic length $l_{m} \equiv(\hbar / e B)^{1 / 2}$ 
(which plays the role of the wavelength in a strong magnetic field $B$ ). In such a smooth potential the quantized cyclotron motion energy $\left(n-\frac{1}{2}\right) \hbar \omega_{c}$ (being the energy of the $n$-th Landau level, $n=1,2, \ldots$ ) is a constant of the motion. The total encrgy $E_{F}$ of an elcctron at the Fermi level is the sum of this Landau level energy and the energy $E_{G}$ from the electrostatic potential,

$$
E_{G}=E_{F}-\left(n-\frac{1}{2}\right) \hbar \omega_{c} .
$$

(The spin-splitting of the Landau levels by the Zeeman encrgy is ignored here, for simplicity.) The constancy of the Landau level index $n$ for smooth $V$ implies that the motion of the electron is along the equipotential $V(x, y)=E_{G}$. Classically, the center of the cyclotron orbit is guided along equipotentials by the combined effects of the Coulomb and Lorentz forces. IIence the name guiding center energy for $E_{G}$. The drift velocity $\mathbf{v}_{\text {drift }}$ of the orbit center (known as the guiding center drift) follows by balancing the Coulomb and Lorentz forces,

$$
\mathbf{v}_{d r i f t}=\frac{1}{e B^{2}} \nabla V \times \mathbf{B} .
$$

The wavefunctions of states at the Fermi level have an appreciable amplitude within $l_{m}$ of the equipotentials at $E_{G}$. One can distinguish between extended statcs near the sample boundaries, and localzzed states encircling potential maxima and minima in the bulk, as illustrated in Fig. 1. The extended states with the same Landau level index $n$ are referred to collectively as the $n-$ th edge channel. The cdge channel with the smallest index $n$ is closest to the sample boundary, because it has the largest $E_{G}$ (eq. (2)). This is scen more clcarly in a cross-sectional plot of $V(x, y)$ (Fig. 2). Notice that if the peaks and dips of the potential in the bulk have amplitudes bclow $\hbar \omega_{c} / 2$, then only states with the highest Landau level index can exist in the bulk at the Fermi level.

The simplicity of the guiding center drift along equipotentials has becn originally used in the percolation theory [20-22] of the QIIE, soon after its experimental discovery [1]. In this theory the existence of edge states is ignored, and the Hall resistance is expressed in terms of properties of extended states in the bulk of the sample. Since in equilibrium all Fermi level states in the bulk are localized in general, the percolation theory requires for its applicability a threshold elcctric ficld to create extended bulk states (it is thus not a linear response theory). A description of the QIIE based on extended edge states and localized bulk states, as in Fig. 1, was first put forward by Halperin [23], and further developed by several authors [12,24-27]. With the exception of Büttilier [12], these authors assume local equilibrium at the edge. In the presence of a chemical potential difference $\delta \mu$ betwcen the edges, each edge channel can be shown to carry a current $(e / h) \delta \mu$, and thus to contribute $e^{2} / h$ to the Hall conductance. The equipartitioning of current among the edge channels is characteristic for a local equilibrium. The total number of edge channels $N$ at the Fermi level is cqual to the number of bulk Landau lcvels below the Fermi level (because of the one-to-one correspondence between edge channels and bulk Landau levels). In this case of local equilibrium one thus has the usual integer QHE, $R_{I I}=h / N e^{2}$, with $R_{I I} \equiv 1 / G_{H}$ the IIall resistance (we disregard for convenience of notation the two-fold spin degencracy of each Landau levcl).

The Hall resistance $R_{H}$ is a four-terminal resistance, meaning that the voltage contacts are distinct from the contacts through which the current is passed. For the twoterminal resistance $R_{2 t}$ the voltage is measured between the current-carrying contacts. In 


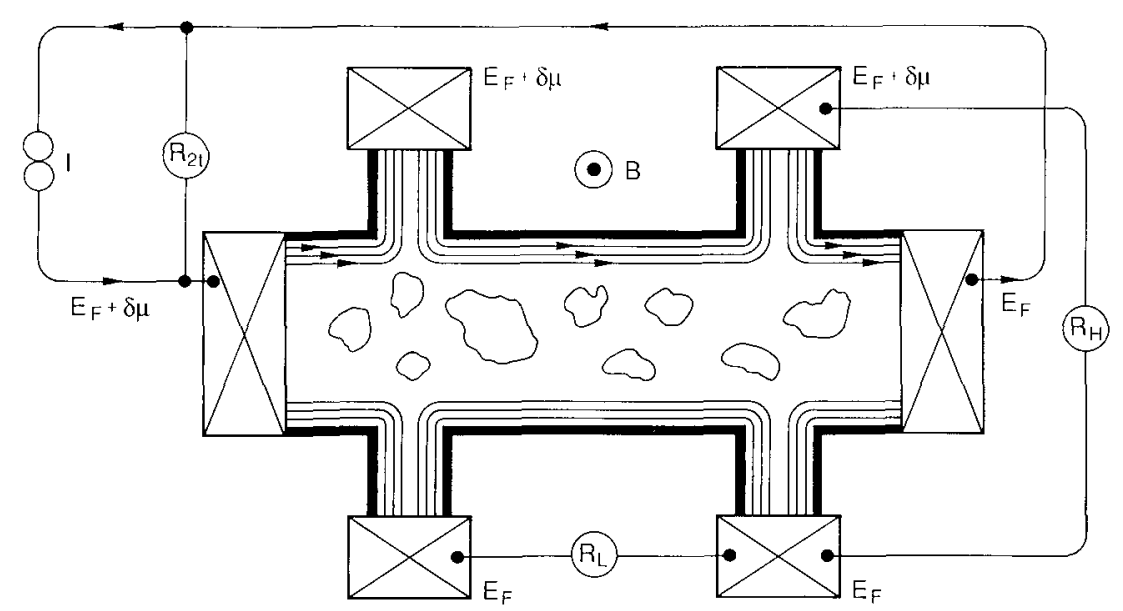

Figure 1. Measurcment configuration for the two-tcrminal resistance $R_{2 t}$, the fourtcrminal IIall resistance $R_{H}$, and the longitudinal resistance $R_{L}$. The edge channels at the Formi level are indicated, arrows point in the dircction of motion of edge channels filled by the source contact at chemical potential $E_{F}+\delta \mu$. The current is cquipartitioned among the edge channels at the upper edge, corresponding to the case of local equilibrium (from Ref. [17]).

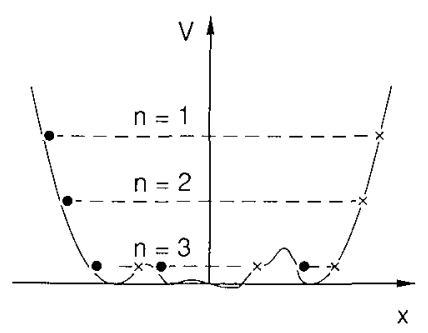

Figure 2. Cross-scction of the elcctrostatic potential $V(x, y)$, along a line perpendicular to the IIall bar in Fig. 1. The location of the states at the Fermi level is indicated by dots and crosses (depending on the dircction of motion). The value of $E_{G}$ for each $n$ is indicated by the dashed line (from Ref. [17]).

the case of local equilibrium these two resistances are the same, $R_{H}=R_{2 t}=h / N e^{2}$, sce Fig. 1. One can also read off from Fig. 1 that the (four-tcrminal) longitudinal resistance $R_{L}$ vanishes, $R_{L}=0$. The distinction between a longitudinal and Iall resistance is topological: A four-terminal resistance measurement gives $R_{I}$ if current and voltage contacts alternate along the boundary of the conductor, and $R_{L}$ if that is not the case. There is no need to further characterize the contacts in the case of local equilibrium at the edge.

If the edges are not in local equilibriurn, the measured resistance depends on the properties of the contacts. Bütliker has devcloped the formalism [12] to treat anomalies in the integer QIIE due to the absence of local equilibrium, when measured with non- 
ideal contacts. To illustrate this formalism we consider, following [17], a situation in which the edge channels at the lower edge are in equilibrium at chemical potential $E_{F}$, while the edge channels at the upper edge are not in local equilibrium. The current at the upper edge is then not equipartitioned among the $N$ cdge channels. Let $f_{n}$ be the fraction of the total current $I$ which is carried by states above $E_{F}$ in the $n$-th edge channel at the upper edge, $I_{n}=f_{n} I$. The voltage contact at the lowcr edge measures a chemical potential $E_{F}$, regardless of its properties. The voltage contact at the upper edge, however, will measure a chemical potential which depends on how it couples to each of the edge channels. The transmission probability $T_{n}$ is the fraction of $I_{n}$ which is 1 ransmitted through the voltage probe to a reservoir at chemical potential $E_{F}+\delta \mu$. The incoming current

$$
I_{\imath n}=\sum_{n=1}^{N} T_{n} f_{n} I, \text { with } \sum_{n=1}^{N} f_{n}=1,
$$

has to be balanced by an outgoing current

$$
I_{\text {out }}=\frac{e}{h} \delta \mu \sum_{n=1}^{N} T_{n}
$$

of equal magnitude, so that the voltage probe draws no net current. (In eq. (5) we have applied a sum rule to identify the total transmission probabilitics of outgoing and incoming edge channcls, see [17].) The requirement $I_{u n}=I_{\text {out }}$ determines $\delta \mu$ and hence the Hall resistance $R_{I I}=\delta \mu / c I$,

$$
R_{I I}=\frac{h}{e^{2}}\left(\sum_{n=1}^{N} T_{n} f_{n}\right)\left(\sum_{n=1}^{N} T_{n}\right)^{-1}
$$

The Hall resistance has its regular quantized value $R_{I I}=h / N e^{2}$ only if eithor $f_{n}=1 / N$ or $T_{n}=1$, for $n=1,2, \ldots N$. The first case corresponds to local equilibrium (the current is cquipartitioncd among the edge channels), the second case to an ideal contact (all edge channels are fully transmitted).

A non-equilibrium population of the edge channcls is generally the result of selective backscattering. Because edgc channels at opposite edges of the sample move in opposite directions, backscattering requires scattcring from one edgc to the other. Selective backscattering of edge channels with $n \geq n_{0}$ is induced by a potential barrier across the sample, if its height is between the guiding center energies of edge channel $n_{0}$ and $n_{0}-1$ (recall that the edge channel with a larger index $n$ has a smaller value of $E_{G}$ ). Selective backscattering can also occur naturally in the absence of an imposed potential barrier. The edge channel with the highest index $n=N$ is selectively backscattcred when the Fermi level approaches the cnergy $\left(N-\frac{1}{2}\right) h \omega_{c}$ of the $N$-th bulk Landau level. The guiding center encrgy of the $N$-th edge channel then approaches zero, and backscattering either by tunneling or by thermally activated processes becomes effective - but for that edge channel only, which remains almost completely decoupled from the other $N-1$ cdge channels over distances as large as $[13,28,29] 250 \mu \mathrm{m}$ (although on that length scale the edge channels with $n \leq N-1$ have cquilibrated to a large cxtent [14]).

We conclude this section by emphasizing that the edge channel formulation of the QIIE by no means implies that the current flows within a few magnctic lengths from the edge. This assumption would be untenable experimentally, see [30]. The flow lines 
in Fig. 1 only show the location of the extended states at the equilibrium Fermi level. A determination of the spatial current distribution, rather than just the total current, requires consideration of all the states below the Formi level, which acquire a net drift velocity because of the Hall field. Within the range of validity of a lincar response theory, however, knowledge of the current distribution is not necessary to know the resistance (see [17] for a further discussion of this point).

\section{Fractional Edge Channels}

In this section we show, following [15], how the concept of an edge channel can be generalized to the fractional QHE, in the case of a smoothly varying clectrostatic potential. This is the case of relevance for experiments on adiabatic transport in the fractional QHE, see section 4. Our result is phrased in terms of a generalized Landaucr formula, in which the cdge channels contribute with a fractional weight. Honce the name "fractional" edge channels. The different generalization of the Landauer formula proposed by MacDonald [16] is discussed in section 5 .

Consider first the equilibrium state of the system. If the clcctrostatic potential energy $V(x, y)$ varies slowly in the $2 \mathrm{DEG}$, then the equilibrium density distribution $n(x, y)$ follows by requiring that the local electrochemical potential $V(\mathrm{r})+\mathrm{d} u / \mathrm{d} n$ has the same value $\mu$ at each point $\mathbf{r}$ in the 2 DEG. Here $\mathrm{d} u / \mathrm{d} n$ is the chemical potential of the uniform $2 \mathrm{DEG}$ with density $n(\mathbf{r})$. It is a remarkable fact $[3,5,6]$ that the inter nal cnergy density $u(n)$ of a uniform interacting $2 \mathrm{DEG}$ in a strong magnetic field has downward cusps at densities $n=\nu_{p} B e / h$ corresponding to certain fractional filling factors $\nu_{p}$. The chemical potential $\mathrm{d} u / \mathrm{d} n$ thus has a discontinuity (an energy gap) at $\nu=\nu_{p}$, with $\mathrm{d} u_{p}^{+} / \mathrm{d} n$ and $\mathrm{d} u_{p}^{-} / \mathrm{d} n$ the two limiting valucs as $\nu \rightarrow u_{p}$. The size of the gap is the cyclotron encrgy $\hbar \omega_{c}$ when $\nu_{p}$ is an integer, and of the order of the Coulomb energy $e^{2} / \varepsilon l_{m}$ when $\nu_{p}$ is a fraction ( $\varepsilon$ is the dielectric constant). An order of magnitude for the energy gap is $10 \mathrm{meV}$ at $B=6 \mathrm{~T}$. As noted by IIalperin [31], when $\mu-V$ lies in the energy gap the filling factor is pinned at the value $\nu_{p}$ :

$$
\begin{aligned}
n= & \nu_{p} B e / h, \text { if } \mathrm{d} u_{p}^{-} / \mathrm{d} n<\mu-V<\mathrm{d} u_{p}^{+} / \mathrm{d} n, \\
& \frac{\mathrm{d} u}{\mathrm{~d} n}+V(\mathbf{r})=\mu, \text { otherwise. }
\end{aligned}
$$

Note that $V(\mathbf{r})$ itself depends on $n(\mathbf{r})$, and thus has to be determined selfconsistently from eq. (7) taling the electrostatic screening in the 2 DEG into account. We do not need to explicitly solve for $n(\mathbf{r})$, but can identify the edge channels from the following general considerations [15].

At the edge of the 2 DEG the clectron density decreases from its bulk value to zcro. Equation (7) implies that this decrease is stepwise, as illustrated in Fig. 3. The requirement on the smoothness of $V$ for the appearance of a well-defined region at the edge in which $\nu$ is pinned at the fractional valuc $\nu_{p}$, is that the change in $V$ within the magnetic length $l_{m}$ is small compared to the energy gap $\mathrm{d} u_{p}^{+} / \mathrm{d} n$ and $\mathrm{d} u_{p}^{-} / \mathrm{d} n$. This ensures that the width of this region is large compared to $l_{m}$, which is a necessary (and presumably sufficient) condition for the formation of the fractional QHE state. Depending on the smoothness of $V$, one thus obtains a series of steps at $\nu=\nu_{p}(p=$ $1,2, \ldots P)$, as one moves from the edge towards the bulk. The series terminates in the filling factor $\nu_{P}=\nu_{b u l k}$ of the bulk, assuming that in the bulk the chemical potential $\mu-V$ lies in an energy gap. The regions of constant $\nu$ at the edge form bands extending 


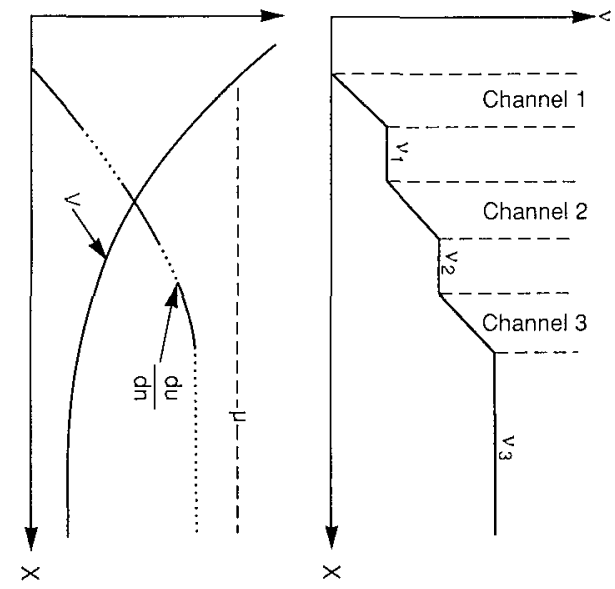

Figure 3. Schemalic drawing of the variation in filling factor $\nu$, clectrostatic potential $V$, and chemical potential $\mathrm{d} u / \mathrm{d} n$, at a smooth boundary in a $2 \mathrm{DEG}$. The dashed line in the bottom pand denotes the constant electrochemical potential $\mu=V+\mathrm{d} u / \mathrm{d} n$. The dotted intervals indicate a discontinuity (energy gap) in $\mathrm{d} u / \mathrm{d} n$, and correspond in the top pancl to regions of constant fractional filling factor $\nu_{p}$ which spatially separate the edge channels. The width of the edge channel regions shrinks to zero in the integer QHE, since the compressibility $\chi$ of these regions is infinitely large in that case (from Ref. [15]).

along the conductor. These incompressible bands (in which the compressibility $\chi \equiv$ $\left.\left(n^{2} \mathrm{~d}^{2} u / \mathrm{d} n^{2}\right)^{-1}=0\right)$ alternate with bands in which $\mu-V$ does not lie in an energy gap. The latter compressible bands (in which $\chi>0$ ) may be identified as the edge channels of the transport problem, as will be discussed below. To resolve a misunderstanding [32], we note that the particular potential and density profilc illustrated in Fig. 3 (in which the edge channels have a non-zcro width) assumes that the compressibility of the edge channels is not infinitely large - but that the analysis given below is independent of this assumption.

The conductance is calculated by bringing onc end of the conductor in contact with a reservoir at a slightly higher electrochcmical potential $\mu+\Delta \mu$. We are concerned with the linear response current, so that the electrostatic potential landscape $V(r)$ is kept at its equilibrium form. The resulting change $\Delta n$ in electron density is

$$
\Delta n=\left.\frac{\delta n}{\delta \mu}\right|_{V} \Delta \mu=-\left.\frac{\delta n}{\delta V}\right|_{\mu} \Delta \mu,
$$

where $\delta$ denotes a functional derivative. In the second equality in eq. (8) it has been used that $n$ is a functional of $\mu-V$, by virtue of eq. (7). In a strong magnetic field, this excess density moves along equipotentials with the guiding-center-drift velocity given by eq. (3). The component $v_{\text {drift }}$ of the drift velocity in the $y$-direction (along the conductor) is

$$
v_{d r i f t}=\hat{\mathbf{y}} \cdot\left(\nabla V \times \mathbf{B} / e B^{2}\right)=-\frac{1}{e B} \frac{\partial V}{\partial x} .
$$


a

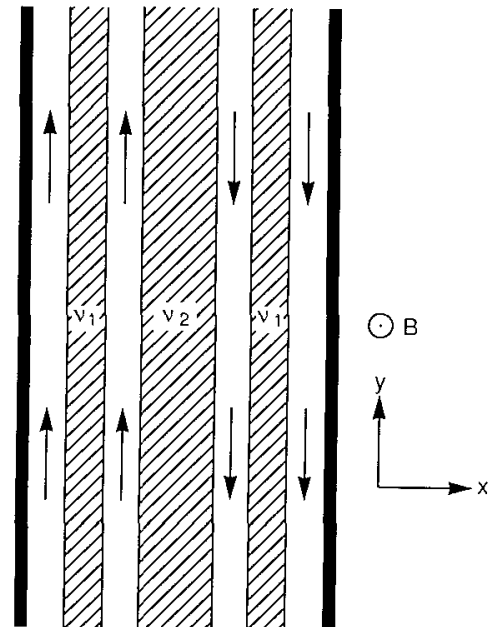

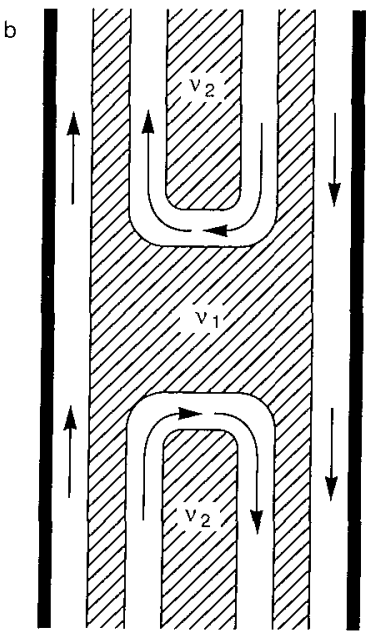

Figure 4. Schematic drawing of the incompressible bands (hatched) of fractional filling factor $\nu_{p}$, alternating with the edge channels (arrows indicate the direction of electron motion in each channel). (a) a uniform conductor; (b) a conductor containing a barrier of reduced filling factor (from Ref. [15]).

The current density $j=-e \Delta n v_{\text {drift }}$ becomes simply

$$
j=-\frac{e}{h} \Delta \mu \frac{\partial \nu}{\partial x}
$$

It follows from eq. (10) that the incompressible bands of constant $\nu=\nu_{p}$ do not contribute to $j$. The reservoir injects the current into the compressible bands at one edge of the conductor only (for which the sign of $\partial \nu / \partial x$ is such that $j$ moves away from the reservoir). The edge channel with index $p=1,2, \ldots P$ is defined as that compressible band which is flanked by incompressible bands at filling factors $\nu_{p}$ and $\nu_{p-1}$. The outermost band from the center of the conductor, which is the $p=1$ edge channel, is included by defining formally $\nu_{0} \equiv 0$. The arrangement of altcrnating edge channcls and compressible bands is illustrated in Fig. 4a. Note that different edges may have a different series of edge channels at the same magnetic ficld value, dcpending on the smoothness of the potential $V$ at the edge (which, as discussed above, determines the incompressible bands that exist at the edge). This is in contrast to the situation in the integer QHE, where a one-to-one correspondence cxists between edge channels and bulk Landau levels (section 2). In the fractional QHE an infinite hierarchy of cnergy gaps exists, in principle, corresponding to an infinite number of possible edge channels - of which only a small number (corresponding to the largest encrgy gaps) will be realized in practice.

The current $I_{p}=(e / h) \Delta \mu\left(\psi_{p}-\nu_{p-1}\right)$ injected into edge channel $p$ by the reservoir follows directly from eq. (10), on integration over $x$. The total current $I$ through the conductor is $I=\sum_{p=1}^{P} I_{p} T_{p}$, if a fraction $T_{p}$ of the injected current $I_{p}$ is transmitted to the reservoir at the other end of the conductor (the remainder returning via the opposite 
edge). For the conductance $G \equiv e I / \Delta \mu$ one thus obtains the generalized Landauer formula for a two-terminal conductor $[15]$

$$
G=\frac{e^{2}}{h} \sum_{p=1}^{P} T_{p} \Delta \nu_{p}
$$

which differs from the usual two-torminal Landauer formula [18] by the presence of the fractional weight factors $\Delta \nu_{p} \equiv \nu_{p}-\nu_{p-1}$. In the integer QHE, $\Delta \nu_{p}=1$ for all $p$, so that eq. (11) reduccs to the Landauer formula with unit weight factors.

A multi-terminal generalization of eq. (11) for a two-terminal conductor is easily constructed, following Büttiker [19]:

$$
\begin{aligned}
& I_{\alpha}=\frac{e}{h} \nu_{\alpha} \mu_{\alpha}-\frac{e}{h} \sum_{\beta} T_{\alpha \beta} \mu_{\beta}, \\
& T_{\alpha \beta}=\sum_{p=1}^{P_{\beta}} T_{p, \alpha \beta} \Delta \nu_{p} .
\end{aligned}
$$

Here $I_{\alpha}$ is the current in lead $\alpha$, connected to a reservoir at electrochemical potential $\mu_{\alpha}$, and with fractional filling factor $\nu_{\alpha}$. Equation (13) defines the transmission probability $T_{\alpha \beta}$ from rescrvoir $\beta$ to reservoir $\alpha$ (or the reflection probability, for $\alpha=\beta$ ), in terms of a sum over the generalized edge channels in lead $\beta$. The contribution from each edge channel $p=1,2, \ldots P_{\beta}$ contains the weight factor $\Delta \nu_{p} \equiv \nu_{p}-\nu_{p-1}$, and the fraction $T_{p, \alpha \beta}$ of the current injected by reservoir $\beta$ into the $p$-th edge channel of lead $\beta$ which reaches reservoir $\alpha$. Apart from the fractional weight factors, the structure of eqs. (12) and (13) is the same as that of the usual Büttiker formula [19].

Applying the generalized Landauer formula eq. (11) to the ideal conductor in Fig. $4 \mathrm{a}$, wherc $T_{p}=1$ for all $p$, one finds the quantized two-terminal conductance

$$
G=\frac{e^{2}}{h} \sum_{p=1}^{P} \Delta \nu_{p}=\frac{e^{2}}{h} \nu_{P} .
$$

The four-terminal Hall conductance $G_{H}$ has the same value, because each cdge is in local equilibrium. In the presence of disorder this edge channel formulation of the fractional QHE is generalized in an analogous way as in the intcger QHE, by including localized states in the bulk. In a smoothly varying disorder potential these localized states take the form of circulating edge channels, as in Fig. 1. In this way the filling factor of the bulk can locally deviate from $\nu_{P}$ without a change in the Hall conductance, leading to the formation of a plateau in the magnetic field dependence of $G_{I}$. In a narrow channel, localized states are not required for a finite plateau width, because the edge channels make it possible for the chemical potential to lie in an energy gap for a finite magnetic field interval. The Hall conductance then remains quantized at $\nu_{P}\left(e^{2} / h\right)$ as long as $\mu-V$ in the bulk lies between $\mathrm{d} u_{P}^{+} / \mathrm{d} n$ and $\mathrm{d} u_{P}^{-} / \mathrm{d} n$. 


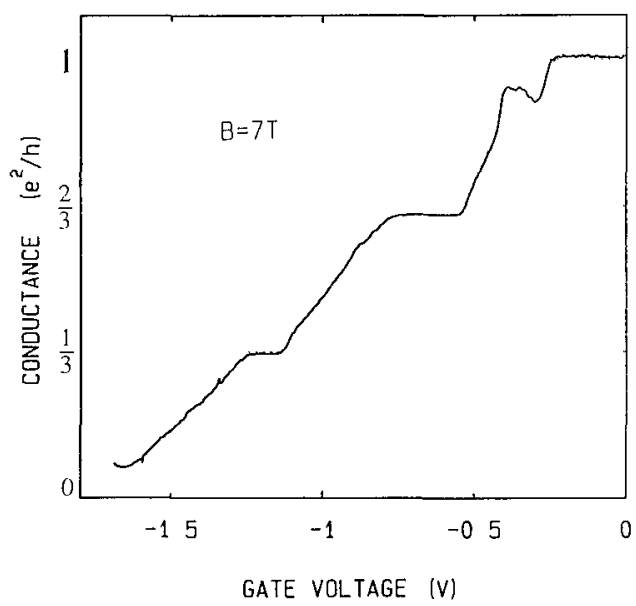

Figure 5. Two-terminal conductance of a constriction containing a potential barrier, as a function of the voltage on the split gate defining the constriction, at a fixed magnetic field of $7 \mathrm{~T}$. The conductance is quantized according to eq. (15) (from Ref. [33]).

\section{Experiments}

We now apply the generalized Landauer formula eq. (11) to some recent experiments on adiabatic transport in the fractional QHE regime. Consider first a conductor containing a potential barrier. The potential barrier corresponds to a region of reduced filling factor $\nu_{P_{\min }} \equiv \nu_{\min }$ separating two regions of filling factor $\nu_{P_{\max }} \equiv \nu_{\max }$. The arrangement of edge channels and incompressible bands is illustrated in Fig. 4b. We assume that the potential barrier is sufficiently smooth that scattering between the edge channels at opposite edges can be neglected. All transmission probabilities are then either zero or one: $T_{p}=1$ for $1 \leq p \leq P_{\min }$ and $T_{p}=0$ for $P_{\min }<p \leq P_{\max }$. Equation (11) then tells us that the two-terminal conductance is

$$
G=\frac{e^{2}}{h} \nu_{\min }
$$

In Fig. 5 we have reproduced experimental data by Kouwenhoven et al. [33] on the fractionally quantized two-terminal conductance of a constriction containing a potential barrier. The constriction (or point contact [29]) is defined by a split gate on top of a GaAs-AlGaAs heterostructure. The conductance in Fig. 5 is shown for a fixed magnetic field of $7 \mathrm{~T}$ as a function of the gate voltage. Increasing the negative gate voltage increases the barrier height, thereby reducing $G$ below the Hall conductance corresponding to $\nu_{\max }=1$ in the wide 2 DEG. The curve in Fig. 5 shows plateaus corresponding to $\nu_{\min }=1,2 / 3$ and $1 / 3$ in eq. (15). The $2 / 3$ plateau is not exactly quantized, but is too low by a few percent. The constriction width on this plateau is estimated [33] at $W=$ $500 \mathrm{~nm}$, which is a factor of 50 larger than the magnetic length at $B=7 \mathrm{~T}$. It would seem that scattering between fractional edge channels at opposite edges (necessary to reduce the conductance below its quantized value) can only occur via states in the bulk for this large ratio of $W / l_{m}$. 
Timp et al. [34] have measured the four-terminal Irall conductance in a narrow cross geometry $(W=90 \mathrm{~nm}$ ). They find, in addition to quantized plateaus near $1 / 3,2 / 5$, and $2 / 3 \times e^{2} / h$, also a plateau-like feature around $1 / 2 \times e^{2} / h$. (This even-denominator fraction is special because it is not observed as a Ilall plateau in a bulk 2 DEG). Notice, however, that the $500 \mathrm{~nm}$ wide constriction of Fig. 5 has a conductance which is featureless at $e^{2} / 2 h$. A narrower constriction $(W=150 \mathrm{~nm})$ studied by Kouwenhoven et al. [33] shows more fluctuations on the plateaus at $1 / 3$ and $2 / 3 \times e^{2} / h$, but no plateau-like feature at $1 / 2 \times e^{2} / h$. The origin of the difference between these two expcriments[33,34] remains to be understood.

A four-terminal measurement of the fractional QIIE in a conductor containing a potential barrier can be analyzed by means of eqs. (12) and (13). The longitudinal resistance $R_{L}$ of the barrier (measured by two adjacent voltage probes, one at each side of the barrier) is given by

$$
R_{\mathrm{L}}=\frac{h}{e^{2}}\left(\frac{1}{\nu_{\min }}-\frac{1}{\nu_{\max }}\right)
$$

This result follows from eqs. (12) and (13) provided enther the edge channcls transmitted across the barrier have equilibrated with the extra edge channels available outside the barrier region; or the voltage contacts are ideal, i.e. they have unit transmission probability for all fractional edge channcls. In the case of the integer QHE, eq. (16) (with $\nu$ integer) was derived some time ago by Van Houten et al. [35] and (independently) by Büttiker [12], and was found to be in agreement with experiments [35-37]. Chang and Cunningham [38] have measured $R_{L}$ in the fractional QIIE, using a $1.5 \mu \mathrm{m}$ wide $2 \mathrm{DEG}$ channel with a gate across a segment of the channel. Contacts to the gated and ungated regions allowed $\nu_{\min }$ and $\nu_{\max }$ to be determined independently. Equation (16) was found to hold to within $0.5 \%$ accuracy.

adiabatic transport in the fractional QHE has been demonstrated [11] by the selective population and detcction of fractional edge channels, achieved by means of barriers in two closely separated current and voltage contacts. The geometry is illustrated in Fig. 6a. It is essentially the same as the geometry employed by Van Wees et al. [10] for the sclective population and detection of Landau levels in the integer QHE. Fig. 6b illustrates the arrangement of fractional edge channels and incompressible bands for the case that the chemical potential lies in an energy gap for the bulk $2 \mathrm{DEG}$ (at $\nu=\nu_{\text {bulk }}$ ), as well as for the two barriers (at $\nu_{I}$ and $\nu_{V}$ for the barrier in the current and voltage lead, respectively). Adiabatic transport is assumed over the barricr, as well as from barrier $I$ to barrier $V$ (for the magnetic field direction indicated in Fig. 6). Equation (12) for this case reduces to

$$
\begin{aligned}
I & =\frac{e}{h} \nu_{I} \mu_{I}, \\
0 & =\frac{e}{h} \nu_{V} \mu_{V}-\frac{e}{h} \min \left(\nu_{I}, \nu_{V}\right) \mu_{I},
\end{aligned}
$$

so that the Hall conductance $G_{\mathrm{H}}=e I / \mu_{\mathrm{V}}$ becomes

$$
G_{H}=\frac{e^{2}}{h} \max \left(\nu_{I}, \nu_{V}\right) \leq \frac{e^{2}}{h} \nu_{\text {bulk }}
$$

The quantized Hall plateaus are determined by the fractional filling factors of the current and voltage leads, not of the bulk 2 DEG. 

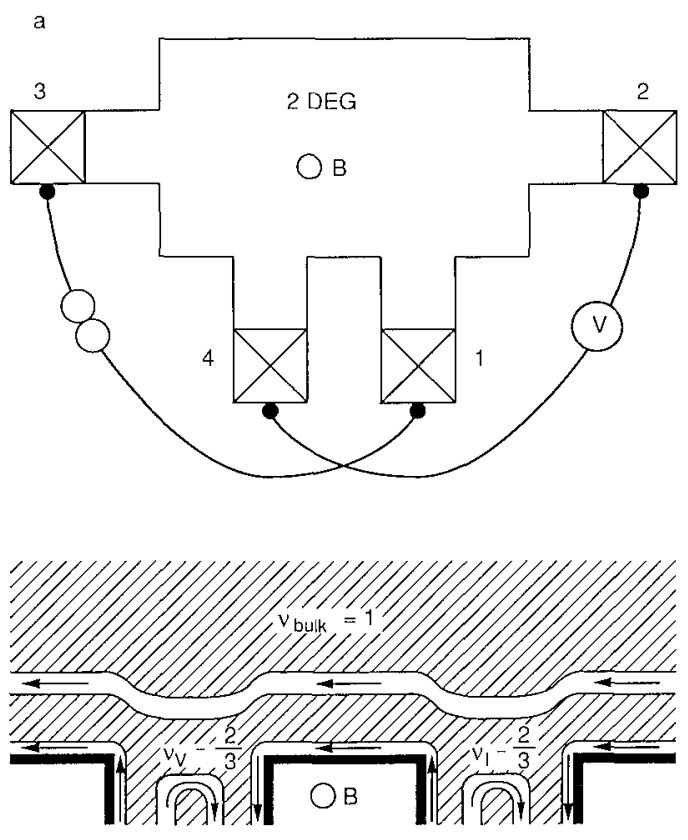

b

Figure 6. (a) Schematic drawing of the experimental geometry of Kouwenhoven et al. [11]. The crossed squares are contacts to the $2 \mathrm{DEG}$. One current lead and one voltage lcad contain a barricr (shaded), of which the hcight can be adjusted by means of a gate (not drawn). The current $I$ flows betwecn contacts 1 and 3 , the voltage $V$ is mcasured between contacts 2 and 4. (b) Arrangement of incompressible bands (hatched) and cdge channels ncar the two barricrs. In the absence of scattcring betwcen the two fractional edge channcls one would measure a IIall conductance $G_{H} \equiv I / V$ which is fractionally quantized at $\frac{2}{3} \times e^{2} / h$ although the bulk has unit filling factor (from Ref. [15]).

Kouwenhoven et al. [11] have demonstrated the sclective population and detection of fractional edge channels in a device with a $2 \mu \mathrm{m}$ scparation of the gates in the current, and voltage leads. The gates extended over a length of $40 \mu \mathrm{m}$ along the 2 DEG boundary. In Fig. 7 wo reproduce one of their expenimental traces. The Hall conductance is shown for a fixed magnetic field of $7.8 \mathrm{~T}$ as a function of the gate voltage (all gates bcing at the same voltage). As the barrier heights in the two leards are increased, the Itall conductance decreases from the bulk value $1 \times e^{2} / h$ to the value $\frac{2}{3} \times e^{2} / h$ det crmined by the lcads - in accord with eq. (18). A more gencral formula for $G_{H}$ valid also in between the quantized plateaus is shown in [11] to be in quantitative agreement with the experiment. 


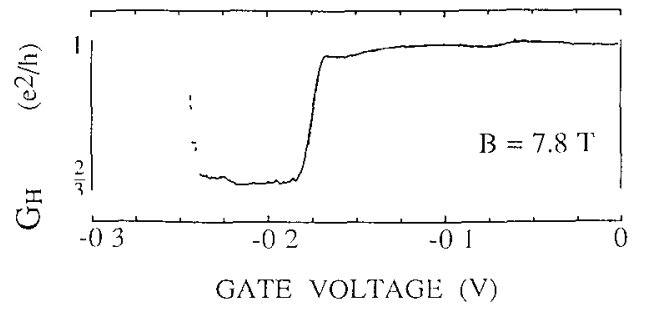

Figure 7. Anomalously quantized IIall conductance in the gcometry of Fig. 6 , in accord with cq. (1S) $\left(\nu_{\text {bulk }}=1, \nu_{I}=\right.$ $\nu_{V}$ decreases from 1 to $2 / 3$ as the negative gate voltage is increased). The temperature is $20 \mathrm{mK}$. The rapidly rising part (dotted) is an artifact due to barrier pinch-off (from Ref. [11]).

\section{Open Problems}

\subsection{What Charge does the Resistance Measure?}

The fractional quantization of the conductance in the expcriments discusscd above is understood as a conscquence of the fractional weight factors in the gencralized Landauer formula cq. (11). These weight factors $\Delta \nu_{p}=\nu_{p}-\nu_{p-1}$ arc not in gencral equal to $e^{*} / e$, with $e^{*}$ the fractional charge of the quasi-particle excitations of Laughlin's incompressible state. The reason for the absence of a onc-to-one correspondence between $\Delta \nu_{p}$ and $e^{*}$ is that the edge channels themselves are not incompressible [15]. The transmission probabilitics in eq. (11) refer to charged "gapless" excitations of the edge channels, which are not identical to the charge $e^{*}$ excitations above the cnergy gap in the incompressible bands (the latter charge might be obtained from thermal activation measurements, see [39]).

It is an interesting and (to date) unsolved problem to determine the charge of the cdge channcl excitations. Kivelson and Polirovsky [40] have suggested performing tunneling expcriments in the fractional QIIE regime for such a purpose, by using the charge dependence of the magnetic length $(\hbar / e B)^{1 / 2}$ (which detcrmines the penetration of the wave function in a tunnel barrier, and hence the transmission probability through the barrier). Altcrnatively, one could use the $h / e$ periodicity of the Aharonov-Bohm magnctoresistance oscillations as a measure of the edgc channcl charge. Simmons et al. [41] find that the characteristic field scale of quasi-periodic resistance fluctuations in a $2 \mu \mathrm{m}$ wide Hall bar increases from $0.016 \mathrm{~T} \pm 30 \%$ ncar $\nu=1,2,3,4$ to $0.05 \mathrm{~T} \pm 30 \%$ near $\nu=\frac{1}{3}$. This is suggestive of a reduction in charge from $e$ to $e / 3$, but not conclusive since the area for the $\Lambda$ haronov-Bohm effect is not well-defined in a Iall bar.

\subsection{Electron and Hole Channels}

MacDonald has, independently of [15], proposed a different generalized Iandauer formula for the fractional QIIE [16] in a smooth electrostatic potential. The difference with eq. (11) is that the weight factors in MacDonald's formula can take on both positive and 
negative values - corresponding to clectron and hole channels, respectively. In the case of local equilibrium at the edge, the sum of weight factors is such that the two formulations give identical results. The results differ in the absence of local equilibrium, if fractional edge channels are selectively populated and detected. For cxample, MacDonald predicts a negatıve longitudinal resistance in a conductor at filling factor $\nu=2 / 3$ containing a segment at $\nu=1$. Another implication of [16], as we understand it, is that the twoterminal conductance $G$ of a conductor at $\nu_{\max }=1$ containing a potential barrier at filling factor $\nu_{\min }$ is reduced to $\frac{1}{3} \times e^{2} / h$ if $\nu_{\mathrm{mm}}=1 / 3$ (in accord with eq. (15)), but remains at $1 \times e^{2} / h$ if $\nu_{\min }=2 / 3$. That this is not observed experimentally (see Fig. 5) could be due to inter-edge channel scattering, as argued by MacDonald. The experiment by Kouwenhoven et al. [11] (Fig. 7), however, is apparently in the adiabatic regime, and was interpreted in Fig. 6 in terms of an edge channel of weight $1 / 3$ at the edge of a conductor at $\nu=1$. In MacDonald's formulation, the conductor at $\nu=1$ has only a single edge channel of weight 1 . This would have to be reconciled with the experimental observation of quantization of the Hall conductance at $2 / 3 \times e^{2} / h$. What is needed is a theory which allows one to introduce edge channels not only for the case of a smooth potential at the edge (considered in [15] and [16]), but also for an abrupt confinement. Such a theory exists for the integer QHE [23] but not yet for the fractional effect.

The presence of both positive and negative weights in a generalized Landauer formula has an interesting implication for the accuracy of the fractional QIIE. As we discussed in section 2 for the integer QHE, accurate quantization of the IIall resistance requires either a local equilibrium at the cdge, or ideal contacts (i.e. contacts which fully transmit all available cdge channcls [12]). The reduction of inter-cdge channel scattering in strong magnetic fields leads to deviations from local equilibrium (i.e. the current is not equipartitioned among the edge channels). Ideal contacts then become necessary for accurate quantization. A contact is esscntially a region with a high clectron density connected to the low-density electron gas, sec Fig. 8a. An ideal contact is realized by a smooth increase in density in the contact region, so that the edge channels in the 2 DEG are transmitted adiabatically into the contact. The contact then induces a local equilibrium by redistributing the current among the edge channcls. This is illustrated in Fig. $8 \mathrm{~b}$ by means of arrows, which indicate the current-carrying edge channels: One incoming edge channel carries the current, whereas both outgoing cdge channels are populated by the contact. These considerations for the integer QHE carry over complctely to the fractional edge channels described in section 3. Ilowever, if both electron and hole channels are present in the $2 \mathrm{DEG}$, then the situation is different. A hole channel is reflected on approaching a region with a smoothly increasing clectron density [16]. In other words, a contact can not be "idcal" (i.e. fully transmitting) for both electron and hole channels. As shown in Fig. 8c, the contact is then not able to redistribute the current among the edge cliannels. The accuracy of the fractional QIIE would thus be limitcd by the extent to which inelastic scattering is effective in establishing a local equilibrium betweon electron and hole channcls - regardless of the idcality of the contacts. This conclusion is of importance not only for adiabatic transport in the fractional QIIE, but for other situations as well in which coexisting electron and hole channels are believed to occur. One example is the integer QHE in a periodic potential discussed by MacDonald in this volume. Another is the integer QHE in parallely conducting electron and hole gases, present in certain semiconductor heterostructures. 


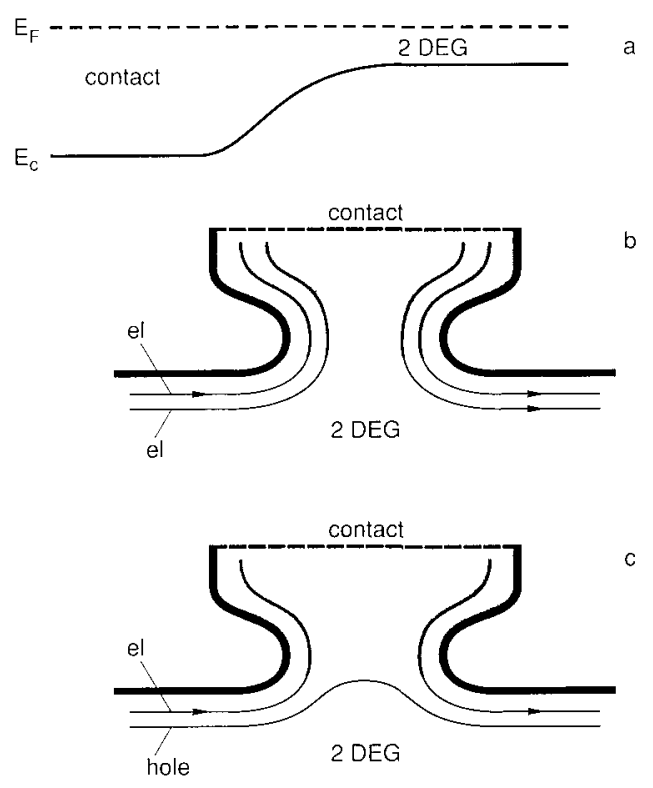

Figure 8. (a) Schematic drawing of the bottom of the conduction band $E_{c}$ and the Fermi encrgy $E_{F}$ at the transition from a low-density to a high-density region in a 2 DEG. (b,c) Top view of a 2DEG near a contact, modeled by a high-density region (shaded) as in (a). The contact is ideal (i.e. fully transmitting) for electron channels (b), but not for hole channcls (c). The arrows indicate the current-carrying cdge channels. This figure illustrates why a contact is effective in cstablishing local equilibrium among clectron channels, but not among electron and hole channcls. In case (c) one would mcasure anomalies in the Hall conductance, due to the absence of local equilibrium.

\section{References}

[1] K. von Klitzing, G. Dorda, and M. Peppcr, Phys. Rev. Lett. 45, 494 (1980)

[2] D.C. Tsui, H.L. Störmer, and A.C. Gossard, Phys. Rev. Lett. 48, 1559 (1982)

[3] R.B. Laughlin, Phys. Rev. Lett. 50, 1395 (1983)

[4] R.B. Laughlin, Phys. Rev.B 27, 3383 (1983)

[5] The Quantum Iall Effect, R.E. Prange and S.M. Girvin, eds., Springer, New York (1987)

[6] T. Chakraborty and P. Pietiläinen, The Fractional Quantum Hall Effect, Springer, Berlin (1988) 
[7] D.A. Wharam, T.J. Thornton, R. Newbury, M. Pepper, H. Ahmed, J.E.F. Frost, D.G. Hasko, D.C. Peacock, D.A. Ritchie, and G.A.C. Jones, J. Phys. C 21, L209 (1988)

[8] B.J. van Wees, L.P. Kouwenhoven, II. van IIouten, C.W.J. Becnakker, J.E. Mooij, C.T. Foxon, and J.J. Harris, Phys. Rev. B 38, 3625 (1988)

[9] A.M. Chang, G. Timp, J.E. Cunningham. P.M. Mankiewich, R.E. Behringer, and R.E. Iloward, Sol. St. Commun. 76, 769 (1988)

[10] B.J. van Wees, E.M.M. Willems, C.J.P.M. IIarmans, C.W.J. Beenakker, H. van Houten, J.G. Williamson, C.T. Foxon, and J.J. Harris, Phys. Rev. Lelt. 62, 1181 (1989)

[11] L.P. Kouwenhoven, B.J. van Wees, N.C. van der Vaart, C.J.P.M. Harmans, C.E. Timmering, and C.T. Foxon, Phys. Rev. Lett. 64, 685 (1990)

[12] M. Büttiker, Phys. Rev. B 38, 9375 (1988)

[13] S. Komiyama, H. Iirai, S. Sasa, and S. Hiyamizu, Phys. Rev. B 40, 12566 (1989)

[14] B.W. Alphenaar, P.L. McEuen, R.G. Whceler, and R.N. Sacks, Phys. Rev. Lett. 64, 677 (1990)

[15] C.W.J. Beenakker, Phys. Rev. Letı. 64, 216 (1990)

[16] A.H. MacDonald, Phys. Rev. Lett. 64, 220 (1990)

[17] C.W.J. Beenakker and II. van IIouten, 'Quantum Transport in Semiconductor Nanostructures', to appear in: Solid State Physics, II. Ehrenreich and D. Turnbull, eds., Academic Press, New York (1991)

[18] R. Landauer, IBM J. Res. Dev. 1 , 223 (1957); ibidem, 32, 306 (1988)

[19] M. Büttiker, Phys. Rev. Jctt. 57, 1761 (1986); IBM J. Res. Dcv. 32, 317 (1988)

[20] R.F. Kazarinov and S. Luryi, Phys. Rev. B 25, 7626 (1982); S. Luryi and R.F. Kazarinov, Phys. Rev. B 27, 1386 (1983); S. Luryi, in: High Magnetic Fields in Semiconductor Physics, G. Landwehr, ed., Springer, Berlin (1987)

[21] S.V. Iordansky, Sol. St. Commun. 43, 1 (1982)

[22] S.A. Trugman, Phys. Rev. B 27, 7539 (1983)

[23] B.I. IIalperin, Phys. Rev. B 25, 2185 (1982)

[24] A.H. MacDonald and P. Streda, Phys. Rev. B 29, 1616 (1984)

[25] S.M. Apenko and Yu.E. Lozovik, J. Phys. C 18, 1197 (1985)

[26] P. Streda, J. Kucera, and A.II. MacDonald, Phys. Rev. Lett. 59, 1973 (1987)

[27] J.K. Jain and S.A. Kivelson, Phys. Rev. B 37, 4276 (1988)

[28] B.J. van Wees, E.M.M. Willems, L.P. Kouwenhoven, C.J.P.M. Harmans, J.G. Williamson, C.T. Foxon, and J.J. IIarris, Phys. Rev. B 39, 8066 (1989)

[29] H. van IIouten, C.W.J. Beenakkcr, and B.J. van Wees, 'Quantum Point Contacts', to appear in: Semiconductors and Scmimetals, M.A. Reed, volume ed., Academic Press, New York (1991)

[30] P.F. Fontein, J.A. Kleinen, P. Ilendriks, F.A.P. Blom, J.H. Wolter, H.G.M. Lochs, F.A.J.M. Driessen, L.J. Giling, and C.W.J. Becnakker, submitted to Phys. Rev. B 
[31] B.I. Halperin, Helv. Phys. Acta 56, 75 (1983)

[32] A.M. Chang, Sol. St. Commun. 74, 871 (1990)

[33] L.P. Kouwenhoven, B.J. van Wees, N.C. van der Vaart, C.J.P.M. Ilarmans, C.E. Timmering, and C.T. Foxon, unpublished

[34] G. Timp, R.E. Behringer, J.E. Cunningham, and R.E. Iloward, Phys. Rev. Lett. 63, 2268 (1989)

[35] II. van Houten, C.W.J. Bccnakker, P.H.M. van Loosdrecht, T.J. Thornton, II. Ahmed, M. Pepper, C.T. Foxon, and J.J. Ilarris, Phys. Rev. B 37, 8534 (1988)

[36] R.J. Ilaug, A.H. MacDonald, P. Streda, and K. von Klitzing, Phys. Rev. Lett. 61, 2797 (1988)

[37] S. Washburn, A.B. Fowler, II. Schmid, and D. Kern, Phys. Rev. Lett. 61, 2801 (1988)

[38] A.M. Chang and J.E. Cunningham, Sol. St. Commun. 72, 651 (1989)

[39] R.G. Clark, J.R. Mallett, S.R. Maynes, J.J. IIary is, and C.T. Foxon, Phys. Rev. Lett. 60, 1747 (1988)

[10] S.A. Kivelson and V.L. Pokrovshy, Phys. Rev. B 40, 1373 (1989)

[41] J.A. Simmons, H.P. Wei, L.W. Engel, D.C. Tsui, and M. Shayegan, Phys. Rev. Lett. $63,1731(1989)$ 